\title{
THE DISTRIBUTION OF DARK MASS IN GALAXIES
}

\author{
Techniques, Puzzles, and Implications for Lensing
}

\author{
PENNY D. SACKETT \\ Kapteyn Astronomical Institute \\ 9700 AV Groningen, The Netherlands
}

\begin{abstract}
Gravitational lensing is one of a number of methods used to probe the distribution of dark mass in the Universe. On galactic scales, complementary techniques include the use of stellar kinematics, the kinematics and morphology of the neutral gas layer, kinematics of satellites, and the morphology and temperature profile of X-ray halos. These methods are compared, with emphasis on their relative strengths and weaknesses in constraining the distribution and extent of dark matter in the Milky Way and other galaxies. It is concluded that (1) the extent of dark halos remains ill-constrained, (2) halos need not be isothermal, and (3) the dark mass is probably quite flattened.
\end{abstract}

\section{Introduction}

Modeling the gravitational structure of a galaxy, and therefore its lensing properties, requires knowledge of the extent, radial profile, and the geometric form of its mass distribution. The interpretation of microlensing rates and optical depths along different lines of sight through the Milky Way, for example, is strongly dependent on the assumed distribution of total (light and dark) Galactic mass. On larger scales, efficient and reliable image-inversion techniques designed to measure the structure parameters of intervening lensing galaxies require appropriate fitting functions for the lensing mass.

This review focuses on techniques that form a symbiotic relationship with lensing in producing valuable and complementary constraints on galactic potentials, especially in providing partial answers to the following questions about galactic dark mass:

- What is the physical extent of dark matter in galaxies?

- Is the distribution of dark mass isothermal? 
- What is the shape of dark "halos"?

\section{How Big are Dark Halos?}

The size of dark halos controls the galactic "sphere of influence" for lensing, interactions, and accretion. Together with the radial and vertical structure parameters, halo extent determines the total mass of the galaxy. The notion of halos as distinct entities ceases to be useful, of course, on scales larger than half the mean distance to the nearest, comparably-sized neighbor.

If halos are extremely large and isothermal, they cannot be totally baryonic without violating the constraints on $\Omega_{B} h^{2}$ from primordial big bang nucleosynthesis (BBN) models. Recent assessments give $0.01 \leq \Omega_{B} \leq 0.06$ for $0.5 \leq h_{100} \leq 1$ (Walker et al. 1991, Smith, Kawano \& Malaney 1993). Since the density of observed baryons is $\Omega_{\text {Lum }} \lesssim 0.007$ (Pagel 1990), the average ratio of dark-to-luminous baryons is $0.4 \leq M_{B \text {,Dark }} / M_{B \text {,Lum }} \leq 8$, and at least some of the Universe's baryons are dark. Rotation curve analysis indicates that $1 \lesssim M_{B \text {,Dark }} / M_{B, \text { Lum }} \lesssim 10$ (cf. Broeils 1992), so that on scales comparable to HI disks ( $\sim 30 \mathrm{kpc})$, halos composed entirely of dark baryons are consistent with BBN. Faint galaxies are more numerous and more dark matter dominated than brighter galaxies, but contribute less to the total luminosity of the Universe. Thus the upper limit placed by BBN on the size of baryonic halos is likely to be considerably larger than $30 \mathrm{kpc}$ (Binney \& Tremaine 1987), but its calculation requires a model-dependent integral over the galaxy luminosity function, weighted by $M_{B, \text { Dark }} / M_{B, \text { Lum. }}$.

\subsection{THE EXTENT AND MASS OF THE MILKY WAY HALO}

At large radius, the mass of our galaxy can be estimated from the kinematics of distant, presumably bound, objects - halos stars, satellite galaxies, and group members - and from the kinematics of the Magellanic Clouds + Stream system. The former has been done most recently by Kochanek (1995), who finds that, using a Jaffe model as the global mass distribution for the Galaxy, the total mass inside $50 \mathrm{kpc}$ at $90 \%$ confidence is $(5.4 \pm 1.3) \times 10^{11} \mathrm{M}_{\odot}$ if the timing constraints of the Local Group are imposed and Leo $\mathrm{I}$ is bound, and somewhat lower at $4.3_{-1.0}^{+1.8} \times 10^{11} \mathrm{M}_{\odot}$ if the timing constraints are not imposed. The corresponding masses within $100 \mathrm{kpc}$ are $7_{-3}^{+4} \times 10^{11} \mathrm{M}_{\odot}$ and $(8 \pm 2) \times 10^{11} \mathrm{M}_{\odot}$, respectively. A recent reexamination of the kinematics and proper motions of the Magellanic Clouds and Stream using two different model potentials for the Milky Way (Lin, Jones \& Kremola 1995) yields $(5.5 \pm 1) \times 10^{11} \mathrm{M}_{\odot}$ inside $100 \mathrm{kpc}$. About one-half this mass must lie outside the present Cloud distance $(50 \mathrm{kpc})$ in order to explain the observed infall of the Magellanic Stream. 
Since the luminous matter in the Galaxy accounts for $(0.6-1) \times 10^{11} \mathrm{M}_{\odot}$, the full range of dark mass estimates from these two methods is $1.3 \times 10^{11} \lesssim$ $M_{\text {Dark }}(<50 \mathrm{kpc}) \lesssim 6.1 \times 10^{11} \mathrm{M}_{\odot}$, with apparent contradictions at the lower end with Local Group timing and at the upper end with Magellanic Stream kinematics. The implied upper limit of $M_{\text {Dark }}(<50 \mathrm{kpc}) \sim 2.7 \times 10^{11} \mathrm{M}_{\odot}$ from the Magellanic Stream model is only just consistent with the lower limit of $\sim 2.3 \times 10^{11} \mathrm{M}_{\odot}$ from the satellite model. For comparison, the spherical isothermal dark halo used by many microlensing teams as a fiducial model contains $4.1 \times 10^{11} \mathrm{M}_{\odot}$ interior to the LMC distance of $50 \mathrm{kpc}$ (Griest 1991). Using this model and its first year of LMC data, the MACHO team concludes with $68 \%$ confidence that the total mass in compact dark lenses is $7_{-4}^{+6} \times 10^{10} \mathrm{M}_{\odot}$ (Alcock et al. 1995). (For complete reviews of the mass of the Galaxy and its dependence on assumptions about Leo I, see Fich \& Tremaine (1991), Schechter (1993), and Freeman (these proceedings).)

\subsection{HALO SIZE OF EXTERNAL GALAXIES}

The kinematics of satellites can also be used to study the halos of external galaxies, but since only a small number of satellites are observed per primary, conclusions are based on a statistical analysis of the sample as a whole. Based on satellite velocities and HI rotation curves, Erickson, Gottesman and Hunter (1987) concluded that the primaries in their sample have $M_{\text {Dark }} / M_{L u m}<5$, total $M / L \sim 20$, and potentials that are well-described by a point mass model - all consistent with dark halos that extend no more than 3 disk radii. In a more recent study using a different sample, however, Zaritsky and White (1994) conclude that halos are nearly isothermal, with total $M(<200 \mathrm{kpc})=1.5-2.6 \times 10^{12} \mathrm{M}_{\odot}$ and $110<M / L<340$ (for $h_{100}=3 / 4$ ). Their result is primarily due to secondaries at 200-300 kpc, where the orbital times are on the order of a Hubble time, thus necessitating the use of halo formation models to interpret the satellite kinematics. Using their method, Zaritsky and White conclude that the Erickson et al. (1987) sample, which has smaller mean primary-satellite separation, is consistent with both small and large mass halos.

In the future, weak lensing is likely to play a larger role in constraining the extent of dark halos. Recent work by Brainerd, Blandford and Smail (these proceedings) has given the first indication that the tangential distortion of background galaxies due to weak lensing by foreground galaxies is statistically measurable given a large sample $(\sim 3000)$ of source-lens pairs. Their measurement of $1.0_{-0.7}^{+1.1} \times 10^{12} h^{-1} \mathrm{M}_{\odot}$ for the total mass within 100 $h^{-1} \mathrm{kpc}$ is consistent both with a mass distribution that grows linearly to $100 \mathrm{kpc}$ and one that truncates much sooner with total $M / L \approx 10$.

The ring of HI gas in the M96 group in Leo (Schneider 1985) offers 
the rare opportunity to sample galactic potentials at very large radii using the well-defined orbits of cold gas. The Leo ring has a radius of $100 \mathrm{kpc}$ and completely encircles the early-type galaxies M105 and NGC 3384. The radial velocities and spatial distribution of the gas are consistent with a single, elliptical Keplerian orbit with a center-of-mass velocity equal to the centroid of the galaxy pair, and a focus that can be placed at the barycenter of the system without compromising the fit. The implied dynamical mass within $100 \mathrm{kpc}$ is $5.6 \times 10^{11} \mathrm{M}_{\odot}$, (only twice that inferred from the internal dynamics of the galaxies), giving a total $M / L \approx 25$. The sensitivity of noncircular orbits to the power law form of the potential suggests that dark matter does not extend much beyond the ring pericenter radius of $60 \mathrm{kpc}$. As a caveat, it is yet clear to what degree M96, a spiral located $60 \mathrm{kpc}$ (in projection) outside the ring, may perturb the ring kinematics.

\section{Are Dark Halos Isothermal?}

The approximate flatness of HI rotation curves is the best observational evidence that dark matter is present in spirals and has a shallower density profile than the light; an isothermal halo is as shallow as $r^{-2}$. Early theoretical studies (Gott 1975) suggested that violent relaxation would cause the inner regions of galaxies to have steeply falling profiles that would flatten to $r^{-2.25}$ in the outer parts. More recent CDM models (Navarro, Frenk \& White 1995) indicate that dark halo profiles may be shallower than $r^{-2}$ in the center and quite steep near the virial radius. Compression by a dissipating gaseous disk may further contract and flatten the dark matter (Blumenthal et al. 1986), accounting in part for the apparent "conspiracy" between the dark and luminous mass that produces flat rotation curves.

\subsection{MILKY WAY}

The radial structure of the mass and light in the Milky Way is less wellconstrained than in external galaxies. Determining the rotation curve of the Galaxy, in particular, has proven notoriously difficult. On the other hand, distances and kinematics of old, resolved stars can be used to measure the vertical restoring force of the local disk - and thus its surface mass density. In this way, Kuijken and Gilmore (1991) report a mass column of $71 \pm 6 \mathrm{M}_{\odot} \mathrm{pc}^{-2}$ within a $1.1 \mathrm{kpc}$ band from the Galactic plane, with $48 \pm$ $9 \mathrm{M}_{\odot} \mathrm{pc}^{-2}$ due to the disk itself, and the rest contributed by a rounder halo. Other recent estimates are similar: Gould (1990) weighs in at $54 \pm 8 \mathrm{M}_{\odot} \mathrm{pc}^{-2}$, Bahcall, Flynn and Gould (1992) at $54 \pm 8 \mathrm{M}_{\odot} \mathrm{pc}^{-2}$, and Flynn and Fuchs (1994) at $52 \pm 13 \mathrm{M}_{\odot} \mathrm{pc}^{-2}$. The dynamical disk mass thus seems to be in remarkable agreement with the detectable disk mass of $49 \pm 9 \mathrm{M}_{\odot} \mathrm{pc}^{-2}-$ at least locally, almost none of the disk mass is dark. Since only about one-half 
of the local rotation support is provided by the observable disk, this further implies that dark matter in the Galaxy is dynamically important at radii as small as 2.5 disk scale lengths. Stated in the language of $\S 3.2$, the Milky Way disk is one-half of its "maximal disk" value. Unfortunately, uncertainties in the outer Galactic rotation curve frustrate attempts to determine the distribution of mass in the outer Galaxy, which is further complicated by a recent suggestion that the generally-accepted local rotation speed, $\Theta_{0}=220$ $\mathrm{km} \mathrm{s}^{-1}$, may be overestimated by $\sim 10 \%$ (Merrifield 1992). A smaller value would increase the relative dynamical importance of the luminous disk and decrease the slope of the outer rotation curve, to which $\Theta_{0}$ is tied.

Conclusions drawn from microlensing results about the dark baryonic content of the Milky Way depend on the assumed distribution of dark and luminous matter in the Galaxy (cf. Paczyński, these proceedings). Many studies have explored how different assumptions for $M / L$, rotation curve slope, and the shape, truncation radius and radial profile of the halo affect these conclusions (cf. references in Griest et al. 1995). As an indication of the importance of luminous structure, lensing by stellar bars in the Milky Way and the LMC has been held accountable, respectively, for most of the optical depth toward the Galactic center (Zhao, Spergel \& Rich 1995) and the LMC (Sahu 1994). On the other hand, if the Galactic disk were "maximal," the MACHO results toward the LMC would be consistent with a dark halo entirely composed of lensing baryons (Alcock et al. 1995).

\subsection{RADIAL DISTRIBUTION OF DARK MASS IN EXTERNAL GALAXIES}

In contrast to the difficulties in the Milky Way, surface brightness profiles and rotation curves for external galaxies can be measured well, but their disk mass-to-light ratios, $M / L$, are uncertain. A disk $M / L$ that is constant with radius (but varies from galaxy to galaxy) can explain the kinematics within the optical radius of many spirals (cf. Kalnajs 1983, Kent 1986, Buchhorn 1992), but the high velocities observed at the edges of HI disks can be reproduced only by invoking a rapid radial increase in $M / L$ (cf. Kent 1987 , Begeman 1987). Since the age and metallicity gradients inferred from the blueing radial color gradients in spirals do not produce these strong, positive gradients in $M / L$ (cf. de Jong 1995), dark matter is implicated.

In order to estimate conservatively the amount of dark matter in a galaxy, the "maximum disk hypothesis" is often adopted (van Albada \& Sancisi 1987), which fixes the disk $M / L$ at the value that maximizes the disk mass without violating kinematic constraints. The hypothesis is controversial (cf. Rubin 1987, Casertano \& van Albada 1990, Freeman 1993), but when it is used to fit rotation curves, the resulting disk $M / L$ are larger for brighter and earlier type spirals than for fainter and later type spirals 

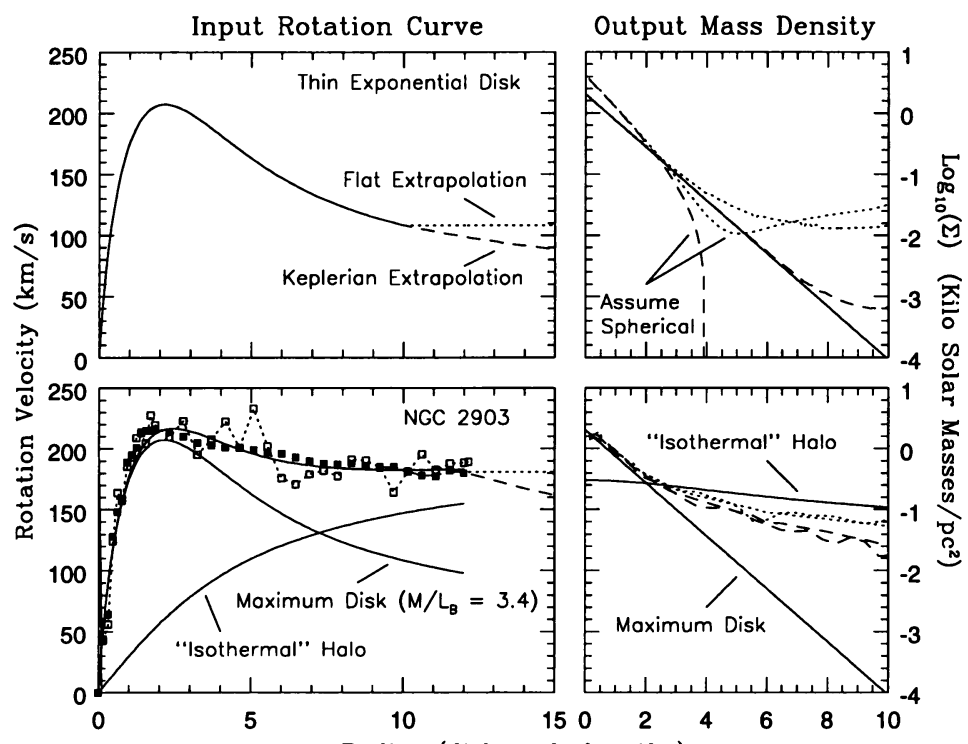

Figure 1. Inversion of rotation curves (left) to to derive dynamical surface mass densities, $\Sigma$ (right). Two extrapolations for $v(r)$ are shown: flat (dotted) and Keplerian (dashed). Top: Thin, exponential disk. Both extrapolations overestimate the true $\Sigma$ (solid) because for an exponential disk $v(r)$ declines faster than Keplerian. Only for inner half of the disk can $\Sigma$ be determined reliably. A spherically-symmetric mass estimator is unreliable for an exponential disk and can produce negative $\Sigma$ in the inversion. Bottom: Sbc NGC 2903. Real data (solid squares) and artificially noisy data (open squares) are inverted using both extrapolation schemes. $M / L$ increases markedly beyond $\sim 2$ scale lengths. Adding noise makes little difference. A spherical isothermal halo has a $\Sigma$ that is $\sim \pi / 2$ larger than that of the flat extrapolation, but with similar slope (Sackett, in preparation).

(Broeils 1992, Buchhorn 1992). The correlation appears to be stronger in bluer bands. These trends may be due to the older stellar populations associated with early spirals, a notion supported by comparison with the $M / L$ derived from stellar population synthesis models (Athanassoula, Bosma \& Papaioannou 1987) and the observed stellar dispersions in spirals (van der Kruit \& Freeman 1986). Alternatively, they may reflect trends in dark matter properties with galaxy type and luminosity that are incorrectly characterized by the application of maximum disk models (van der Kruit 1995).

It should be stressed that rotation curves do not constrain the dark matter distribution to have a $r^{-2}$ (isothermal) volume density profile. If (1) rotation curves were perfectly flat, (2) halos were spherical, and (3) the luminous mass were negligible, then indeed dark matter halos could be described by singular isothermal spheres over the radial range of the kinematics. In fact, rotation curves are seldom flat, but instead have slopes that are systematically related to the peak speed or the luminosity concentration of the galaxy (Kent 1987, Athanassoula, Bosma \& Papaioannou 1987, Casertano \& van Gorkom 1991, Broeils 1992): diffuse, slow rotators have rising rotation curves, while compact, fast rotators have falling curves. 
Evidence is mounting that dark halos are not spherical (§4). Finally, since the stellar mass is not strongly constrained, dark halos with asymptotic $r^{-3}$ and $r^{-4}$ density profiles are also consistent with observed rotation curves (Lake \& Feinswog 1989). Even when $r^{-2}$ halos are used to fit rotation curves - together with luminous disks of reasonable $M / L$ - a large core radius must be assumed, so that the halo does not achieve its asymptotic (isothermal) speed at the last measured point (Fig. 1). This is especially true of maximum disk fits (cf. Broeils 1992), but often applies to fits that assume smaller disk masses as well (cf. Kent 1987). This suggests that the linewidth of the HI gas used in the Tully-Fisher relation is probably not governed by the asymptotic speed of an isothermal dark halo.

Rotation curve inversion is a step toward a model-independent method for determining the radial distribution of dark mass in galaxies. The technique has been criticized as being sensitive to noise (Binney \& Tremaine 1987), but for this application the typical uncertainties of $10-20 \%$ are quite tolerable. The method does depend on the assumed geometry of the mass and extrapolation of the rotation curve beyond the last measured point (Fig. 1), but has the advantage of making this dependence explicit rather than camouflaging it by the use of a particular model for the dark mass.

\section{What is the Shape of the Dark Mass: Disks or Halos?}

Use of term "halo" to describe the distribution of dark matter may be prejudicial: there is no strong theoretical or observational evidence to indicate that dark matter in galaxies is distributed spherically. Dark halos have been favored over dark disks as a means to stabilize galaxies against bar formation (Ostriker \& Peebles 1973), but bulges (Kalnajs 1987) and hot disks (Athanassoula \& Sellwood 1987) are now believed to be more efficient stabilizers. Traditional rotation curve analysis is insensitive to vertical structure, but accumulating observational evidence from other methods suggests that the dark mass may be considerably flattened toward the stellar plane, while remaining relatively axisymmetric, with an in-plane axis ratio of $(b / a)_{\rho}>0.7$ (see review by Rix 1995).

Here, we focus on $(c / a)_{\rho}$, the vertical flattening of dark matter, since it is likely to have the stronger implications for both microlensing in the Galaxy (cf. Gould, Miralda-Escudé, \& Bahcall 1994), and the use of macrolensing as a probe of galaxy structure. The flattening of the dark mass may also provide a clue as to the nature of its constituents. N-body simulations of dissipationless collapse produce strongly triaxial dark halos (Frenk et al. 1988, Dubinski \& Carlberg 1991, Warren et al. 1992), but adding a small fraction $(\sim 10 \%)$ of dissipative gas results in halos of a more consistent shape - nearly oblate, $(b / a)_{\rho} \gtrsim 0.8$, but moderately flattened, $(c / a)_{\rho} \gtrsim 0.6$ 
(Katz \& Gunn 1991, Dubinski 1994). Thus strongly flattened halos, with $(c / a)_{\rho}<0.5$, may imply that dissipation has played an even greater role, perhaps implicating baryonic dark matter.

In order to measure $(c / a)_{\rho}$ of the dark mass, a probe of the vertical gradient of the potential is required. In the Milky Way, the measured anisotropy of the velocity dispersion of extreme Population II halo stars has been used to estimate the flattening of the mass distribution (Binney, May \& Ostriker 1987, van der Marel 1991). Unfortunately, the results depend on the unknown orbital structure of the stellar halo, so that $(c / a)_{\rho}$ can be confined only to lie between 0.3 and 1 at the solar neighborhood.

In external galaxies, Buote and Canizares $(1994,1995)$ have used the flattening of extended X-ray isophotes, assuming that the gas is in hydrostatic equilibrium, to place constraints on the flattening of the dark matter in two early-type systems. For the elliptical NGC 720, they find that the dark isodensity contours have axis ratio $0.3 \lesssim(c / a)_{\rho} \lesssim 0.5$ at $90 \%$ confidence; for the lenticular NGC $1332,0.2 \lesssim(c / a)_{\rho} \lesssim 0.7$. This suggests that these dark halos are at least as flattened as their corresponding luminous galaxies, which have optical isophotes of axis ratio $q \approx 0.6$.

These values contrast with that of $(c / a)_{\rho} \geq 0.84$ derived for the S0 NGC 4753 by Steiman-Cameron, Kormendy \& Durisen (1992) on the basis of fitting an inclined, precessing disk model to the complicated pattern of the galaxy's dust lanes. Their remarkably good fit is independent of $(c / a)_{\rho}$; the flattening constraints are based on the assumption that the gas is smoothly distributed and has completed at least 6 orbits at all radii.

Stable rings around galaxies are not observed to have random orientations, but are found preferentially close to the equatorial or polar planes, suggesting that the potential may be oblate. In particular, polar ring galaxies (PRGs) are surrounded by rings of gas and stars in orbits nearly perpendicular to the central stellar plane; these rings can extend to 20 disk scale lengths. Since in an oblate potential closed ring orbits are elongated along the polar axis and have speeds that vary with ring azimuth, the shape and kinematics of a polar ring are excellent extended probes of $(c / a)_{\rho}$. Early kinematic analyses of three PRGs produced axes ratios for the potentials of $0.86<(c / a)_{\Phi}<1.05$ with uncertainties of 0.2 (Schweizer, Whitmore \& Rubin 1983, Whitmore, McElroy \& Schweizer 1987), corresponding to $0.58 \lesssim(c / a)_{\rho} \lesssim 1.15$ with very large uncertainties. Subsequent studies using more detailed mass models and higher quality data over a larger radial range have narrowed the range for the dark mass to $0.3 \lesssim(c / a)_{\rho} \lesssim 0.6$ (Arnaboldi et al. 1993, Sackett et al. 1994, Sackett \& Pogge 1995); in each galaxy, $(c / a)_{\rho}$ is similar to the inferred flattening of the central stellar body.

Measurements of $(c / a)_{\rho}$ for spiral galaxies are rarer, more difficult, and sorely needed. Assuming that gas disks evolve gravitationally toward a 
discrete bending mode in tilted rigid halos, Hofner and Sparke (1994) find that moderate halo flattening of $0.6 \lesssim(c / a)_{\rho} \lesssim 0.9$ can reproduce the observed HI warps of five spirals. In principle, $(c / a)_{\rho}$ can also be constrained by the flaring of the HI layer; in the most detailed study of this type, Olling and van Gorkom (1995) obtain $0.2<(c / a)_{\rho}<0.8$ for the dark halo of the Sc NGC 4244. Since non-gravitational energy sources may be responsible for a substantial fraction of the vertical support of gas (Malhotra 1995, and references therein), this measurement may be an upper limit to $(c / a)_{\rho}$.

\section{Parting Caveats and a Puzzle}

Since the mass distribution in cluster galaxies may be modified by the interactions and violent relaxation that shape the evolving cluster potential, we have restricted this review to relatively isolated galaxies that are more likely to be dynamically relaxed. Furthermore, we have largely ignored ellipticals, the inner few kpc of which are thought to be responsible for the strong lensing of distant QSOs and radio sources. Although selection effects operate to favor flattened lenses in multiply-imaged systems (Kassiola \& Kovner 1993), image inversion techniques yield lenses that are surprisingly flattened (Kochanek 1995a, and references therein) - the projected $(c / a)_{\Phi} \lesssim 0.8$ corresponds to $(c / a)_{\rho} \lesssim 0.4$. Can these flat lenses be reconciled with the axis ratio distribution of ellipticals, which peaks at $q=0.7$ (Ryden 1992), or are disk galaxies implicated?

Acknowledgements: P.D.S. gratefully acknowledges travel support from the Leids Kerkhoven-Bosscha Fonds and the IAU.

\section{References}

Alcock et al. 1995, ApJ, submitted (astro-ph/9506113)

Athanassoula, E., Bosma, A. \& Papaioannou, S. 1987, A\&A, 179, 23

Athanassoula, E. \& Sellwood, J. A. 1987, IAU Sym. 117, eds. J. Kormendy \& G. R. Knapp (Dordrecht: Reidel), 300

Bahcall, J.N., Flynn, C. \& Gould, A. 1992, ApJ, 389, 234

Begeman, K. 1987, Ph. D. thesis, University of Groningen The Netherlands

Binney, J. \& Tremaine, S. 1987, Galactic Dynamics (Princeton:Princeton Univ. Press)

Binney, J, May, A. \& Ostriker, J.P. 1987, MNRAS, 226, 149

Blumenthal, G. R., Faber, S. M., Flores, R. \& Primack, J. 1986, ApJ, 301, 27

Broeils, A. 1992, Ph. D. thesis, University of Groningen, The Netherlands

Buchhorn, M. 1992, Ph. D. thesis, Australian National University

Buote, D.A. \& Canizares, C.R. 1994, ApJ, 427, 86

Buote, D.A. \& Canizares, C.R. 1995, to appear in ApJ, astro-ph/9508005

Casertano, S. \& van Albada, T.S. 1990, Baryonic Dark Matter, (Dordrecht:Kluwer) 159

Casertano, S. \& van Gorkom J.H. 1991, AJ, 101, 1231

de Jong, R.S. 1995, Ph. D. thesis, University of Groningen, The Netherlands

Dubinski, J., 1994, ApJ, 431, 617

Dubinski, J. \& Carlberg, R., 1991, ApJ, 378, 496

Erickson L.K., Gottesman, S.T. \& Hunter, J.H., 1987, Nature, 325, 779 
Fich, M. \& Tremaine, S., 1991, ARA\&A, 29, 409

Flynn, C. \& Fuchs, B., 1994, MNRAS, 270, 471

Freeman, K.C., 1993, in Physics of Nearby Galaxies: Nature or Nurture?, eds. R.X.

Thuan, C. Balkowski \& J. Thanh Van, (Editions Frontières) 201

Frenk, C.S., White, S.D.M, Davis, M. \& Efstathiou, G., 1988, ApJ, 327, 507

Gould, A., 1990, MNRAS, 244, 25

Gould, A., Miralda-Escudé, J., \& Bahcall, J.N., 1994, ApJL, 423, L105

Griest, K., 1991, ApJ, 366, 412

Griest, K., et al., 1995, in Pascos/Hopkins Symposium, in press (astro-ph/9506016)

Gott, J. R., 1975, ApJ, 201, 296

Hofner, P. \& Sparke, L.S., 1994, ApJ, 428, 466

Kalnajs, A.J. 1983, in IAU Sym. 100, ed. E. Athanassoula (Dordrecht: Reidel) 87

Kalnajs, A.J., 1987, in IAU Sym. 117, eds. J. Kormendy \& G. Knapp (Dordrecht: Reidel) 289

Katz, N. \& Gunn, J. E., 1991, ApJ, 377, 365

Kent, S.M., 1986, AJ, 91, 1301

Kent, S.M., 1987, AJ, 93, 816

Kochanek, C.S., 1995a, ApJ, 445, 559

Kochanek, C.S., 1995b, ApJ, in press

Kassiola, A. \& Kovner, I., 1993, ApJ, 417, 450

Kuijken, K. \& Gilmore, G., 1991, ApJ, 367, 9

Lake, G. \& Feinswog, L., 1989, AJ, 98, 166

Lin, D.N.C., Jones, B.F. \& Klemola, A.R., 1995, ApJ, 439, 652

Malhotra, S., 1995, ApJ, 448, 138

Merrifield, M.R., 1992, AJ, 103, 1552

Navarro, J.F., Frenk, C.S. \& White, S.D.M., 1995, ApJ, submitted (astro-ph/9508025)

Olling, R.P. \& van Gorkom, J.H., 1995, in Dark Matter, eds. S. Holt \& C. Bennett (New York: AIP) 121

Ostriker, J.P. \& Peebles, P.J.E., 1973, ApJ, 186, 467

Pagel, B., 1990, Baryonic Dark Matter, (Dordrecht: Kluwer) 237

Rix, H.-W., 1995, in IAU Sym. 169, in press (astro-ph/9501068)

Rubin, V.C., 1987, in IAU Sym. 117, eds. J. Kormendy \& G. Knapp (Dordrecht: Reidel) 51

Ryden, B.S., 1992, ApJ, 396, 445

Sackett, P.D, Rix, H.-W., Jarvis, B.J., \& Freeman, K.C., 1994, ApJ, 436, 629

Sackett, P.D. \& Pogge, R.W., 1995, in Dark Matter, eds. S. Holt \& C. Bennett (New York: AIP) 141

Sahu, K.C., 1994, Nature, 370, 275

Schechter, P., 1993, in Back to the Galaxy, eds. S. Holt \& F. Verter (New York: AIP) 571

Schneider, S.E., 1985, ApJ, 288, L33

Schweizer, F., Whitmore, B.C. \& Rubin, V.C., 1983, AJ, 88, 909

Smith, M.S., Kawano, L.H. \& Malaney, R.A., 1993, ApJS, 85, 219

Steiman-Cameron, T.Y., Kormendy, J. \& Durisen, R.H., 1992, AJ, 104, 1339

van Albada, T. \& Sancisi, R., 1987 in IAU Sym. 117, eds. J. Kormendy \& G. Knapp

(Dordrecht: Reidel) 67

van der Kruit, P. C. \& Freeman, K. C., 1986, ApJ, 303, 556

van der Kruit, P.C., 1995, in IAU Sym. 164, eds. P.C. van der Kruit \& G. Gilmore (Dordrecht: Kluwer) 205

van der Marel, R.P., 1991, MNRAS, 248, 515

Walker, T., Steigman, G., Kang, H. Schramm, D., \& Olive, K., 1991, ApJ, 376, 51.

Warren, M. S., Quinn, P. J., Salmon, J. K., \& Zurek, W. H., 1992, ApJ, 399, 405

Whitmore, B.C., McElroy, D., \& Schweizer, F., 1987, ApJ, 314, 439

Zaritsky, D. \& White, S. D. M., 1994, ApJ, 435, 599

Zhao, H.-S., Spergel, D. N. \& Rich, R. M., 1995, ApJ, 420, 806 